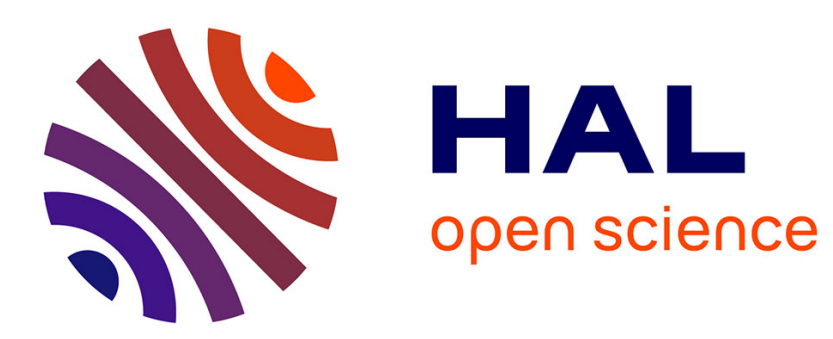

\title{
Two-dimensional noise-sustained structures in optics: Theory and experiments
}

Gonzague Agez, Pierre Glorieux, M. Taki, E. Louvergneaux

\section{To cite this version:}

Gonzague Agez, Pierre Glorieux, M. Taki, E. Louvergneaux. Two-dimensional noise-sustained structures in optics: Theory and experiments. Physical Review A : Atomic, molecular, and optical physics [1990-2015], 2006, 74 (4), 10.1103/PhysRevA.74.043814 . hal-01730642

\section{HAL Id: hal-01730642 \\ https://hal.science/hal-01730642}

Submitted on 13 Mar 2018

HAL is a multi-disciplinary open access archive for the deposit and dissemination of scientific research documents, whether they are published or not. The documents may come from teaching and research institutions in France or abroad, or from public or private research centers.
L'archive ouverte pluridisciplinaire HAL, est destinée au dépôt et à la diffusion de documents scientifiques de niveau recherche, publiés ou non, émanant des établissements d'enseignement et de recherche français ou étrangers, des laboratoires publics ou privés. 


\title{
Two-dimensional noise-sustained structures in optics: Theory and experiments
}

\author{
G. Agez, P. Glorieux, M. Taki, and E. Louvergneaux* \\ Laboratoire de Physique des Lasers, Atomes et Molécules, UMR CNRS 8523, Centre d'Études et de Recherches Lasers et Applications, \\ Université des Sciences et Technologies de Lille, 59655 Villeneuve d'Ascq Cedex, France
}

(Received 11 April 2006; published 19 October 2006)

\begin{abstract}
Translational transverse shifts drastically affect pattern formation in a noisy system with optical feedback. These strong nonlocal interactions may give rise to large domains of convective instability resulting in various types of two-dimensional (2D) noise-sustained structures. These "basic patterns" are investigated and their thresholds and properties are analytically derived. Corresponding 2D experimental patterns are shown to be in complete agreement with theory. Surprisingly enough, some patterns that are purely sustained by noise are found to be nondrifting in contrast with the commonly widespread situation in which convective instabilities lead to traveling patterns.
\end{abstract}

DOI: 10.1103/PhysRevA.74.043814

Pattern formation always occurs through the spontaneous symmetry breaking of a ground state. Just above the threshold corresponding to this symmetry breaking, only perturbations at a critical wavelength are destabilized and expand through the whole transverse space, giving rise to a variety of patterns. In the standard analytical approach of spatial instabilities, the critical wave number that is destabilized at threshold and its associated growth rate are determined, but little attention is paid to the question of the emergence mechanisms and of the transverse spreading of the pattern. Indeed, whether it occurs globally everywhere in the system or it rises up locally and then invades the system by different propagation or amplification processes is an important issue. This inherent question of spatiotemporal evolution of localized perturbations and the subsequent aspects of propagation is crucial in extended transverse systems with a transverse asymmetric nonlocal interaction. This is the case of convective systems where the reflection symmetry is broken and patterns may drift at the onset of instabilities. In such systems, beyond translational symmetry breaking (or first threshold), an initial local perturbation at a critical wavelength is advected away by the nonlocal interaction as it simultaneously grows. Then, two cases are observed: (i) the advection is "faster" than the growth of the initial local disturbance so that the system returns locally to its initial homogeneous equilibrium state; (ii) the growth dominates the drift upstream so that the system reaches a patterned state. The first regime reveals the occurrence of a convective instability $(\mathrm{CI})$ and the second one characterizes the transition to an absolute instability (AI). The two associated final states obtained at long time are the homogeneous state and the patterned one, respectively. This difference drastically changes as soon as noise is present in the system [1]. In the case of the convective regime, the noise acts as a continuous microscopic perturbation source that is selectively spatially amplified to give rise to the noise-sustained pattern. Namely, a noise-sustained structure (NSS) is observed for the convective regime and a self-sustained pattern for the absolute re-

*Electronic address: eric.louvergneaux@univ-lille1.fr; URL: http: //www.phlam.univ-lille1.fr/perso/louvergneaux gime. Thus, a structured state is observed for both regimes with no simple distinction between them in terms of their spatiotemporal evolution.

The concepts of convective and absolute instabilities were first developed in the context of plasma physics [2], and later successfully used in hydrodynamics [3]. So far, convective instabilities leading to noise-sustained structures were theoretically predicted in such diverse fields as open flows [4], optics [5], traffic flow [6], and crystal growth [7]. On the experimental side, noise-sustained structures were obtained in hydrodynamics [8-10] and later in a spatially extended one-dimensional (1D) optical system [1]. In contrast with 1D systems where the advection does not change the nature of the pattern (rolls), in 2D systems the dynamics is richer in term of new patterns, symmetries, wave-vector selection with respect to the drift direction, etc. Moreover, most experiments have been achieved in 1D systems and, as far as we know, NSS have not yet been experimentally evidenced in a $2 \mathrm{D}$ system. The purpose of this paper is to investigate theoretically and experimentally 2D noise-sustained pattern formation in an optical system with an asymmetric nonlocal interaction. The system considered here is a Kerr slice medium with optical feedback where the backward beam is shifted transversely $[11,12]$. It allows us to generate and observe different types of basic 2D experimental noisesustained structures that are destabilized from the homogeneous state at the first convective threshold.

The paper is organized as follows. Section I briefly recalls the main concepts peculiar to convective and absolute instabilities. The model in the presence of a translational displacement and its associated dispersion relation are given in Sec. II. The analytical investigations and predictions, in the ideal situation in which noise is neglected and the incident wave is a plane wave, of all the different types of possible basic "convective modes," their associated thresholds and properties are summarized in Sec. III. Then, the experimentally expected NSS are obtained from numerical simulations carried out in the experimental conditions. In Sec. IV, we determine the absolute thresholds that are compared afterwards with the convective ones found in Sec. III. This allows us to delimit the regions of purely convective modes. Experimental findings are then compared with analytical predictions in 
(a)

Stable

state (b)

Convective threshold (c)

Convective (d)

Absolute threshold (e)

Absolute instability
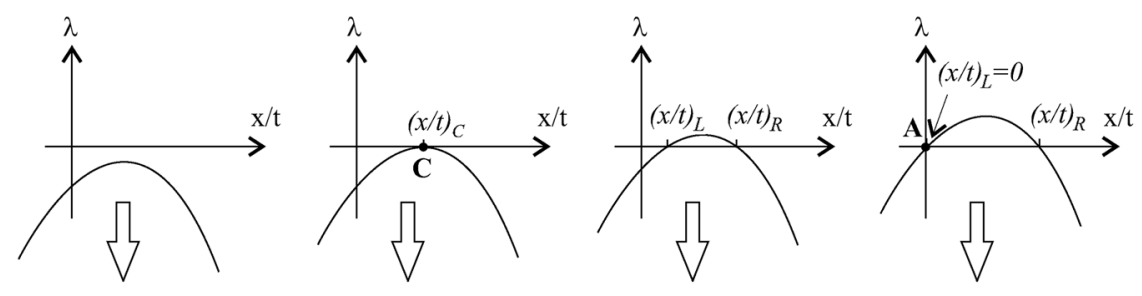

$\lambda$
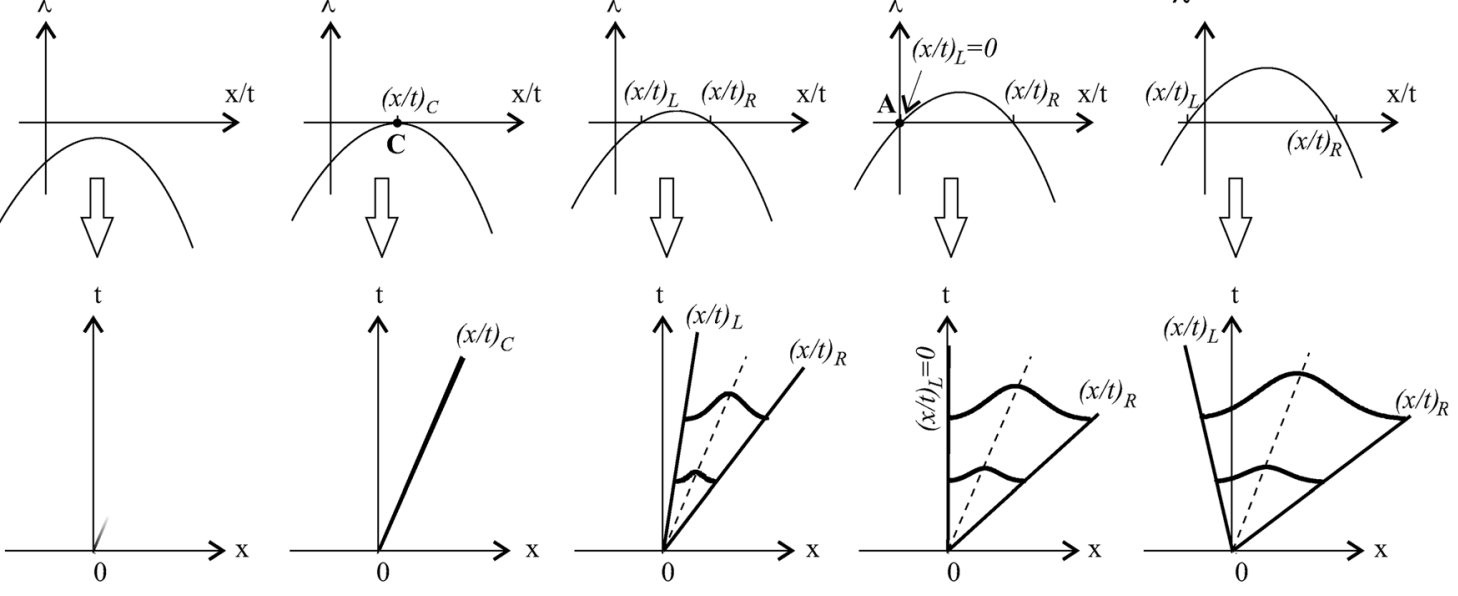

transverse displacement

FIG. 1. Schematic diagram of the different types of wave-packet time evolution from an initial perturbation localized at the origin for the (a) stable, (c) convectively unstable, and (e) absolute unstable regimes in a 1D configuration. The cases (b) and (d) correspond to the thresholds of the last two regimes. The control parameter is increased from (a) to (e). $\lambda$ is the growth rate of the wave packets propagating at velocities $x / t$ along the rays $(x / t)$ in the spatiotemporal diagrams $(x, t)$. The convective threshold is observed when the global maximum of the growth rate reaches zero [point $C$ of case (b)] whereas the absolute threshold is reached when the growth rate of the wave packet having a vanishing group velocity reaches zero [point $A$ of case (d)]. $(x / t)_{L}$ and $(x / t)_{R}$ correspond to the slow and fast frontiers of the wave packets defined by $\lambda\left((x / t)_{L}\right.$ or $\left.R\right)=0$, which are the front solutions limiting the wave packets.

Sec. V, showing excellent agreement. Concluding remarks are given in the final section.

\section{CONVECTIVE AND ABSOLUTE INSTABILITIES}

The standard theoretical approach of the convective and absolute instabilities consists in finding the response of the system to an initial localized perturbation and is provided by the Green function $G(\mathbf{r}, t)[3,13]$, where $\mathbf{r}=(x, y)$ represents the spatial coordinate in the transverse plane. The formal expression of $G(\mathbf{r}, t)$ is an integral over a finite band of wave vectors $\mathbf{k}=\left(k_{x}, k_{y}\right)$ that can rarely be calculated. However, its evolution over long times can be evaluated by the steepestdescent method [14]. In that case, the response of the system to a local perturbation is asymptotically determined by the value of $G(\mathbf{r}, t)$ estimated at the complex wave vector $\mathbf{k}^{*}$ corresponding to a saddle point of the underlying linear dispersion relation. The dominant wave packet, propagating at group velocity $(\mathbf{r} / t)$, that corresponds to the wave vector $\mathbf{k}^{*}$ is defined by

$$
\left.\frac{\partial \Omega(\mathbf{k})}{\partial \mathbf{k}}\right|_{\mathbf{k}^{*}}=\frac{\mathbf{r}}{t},
$$

where $\Omega(\mathbf{k})$ is the complex frequency of linear perturbations satisfying the dispersion relation. The set of rays $(\mathbf{r} / t)$ corresponding to asymptotically diverging values of $G(\mathbf{r}, t)$ defines a wave packet, originating from the initial localized perturbation and propagating in the spatiotemporal domain $(\mathbf{r}, t)$ (Fig. 1). Its evolution provides us with the nature, either convective or absolute, of the instability. In the former case, the wave packet spreads slower than the drift so that it disappears at long times (convective regime) [Fig. 1(c)]. In the second case, the wave packet spread is faster than its advection so that a pattern invades the whole transverse space (absolute regime) [Fig. 1(e)].

The classification of the wave-packet asymptotic evolutions can still be made by the study of the (temporal and spatial) growth rate $\lambda(\mathbf{r} / t)$ (Fig. 1) of the destabilized wave vectors associated with each ray $(\mathbf{r} / t)$ in the packet that constitutes the perturbation $\Delta n(\mathbf{r}, t)$,

$$
\begin{aligned}
\Delta n(\mathbf{r}, t) & \sim e^{i[\mathbf{k} \cdot \mathbf{r}-\Omega(\mathbf{k}) t]}=e^{\left[-\mathbf{k}^{i} \cdot \mathbf{r} / t+\Omega^{i}(\mathbf{k})\right] t} e^{i\left[\mathbf{k}^{r} \cdot \mathbf{r}-\Omega^{r}(\mathbf{k}) t\right]} \\
& =e^{\lambda(\mathbf{r} / t) t} e^{i\left[k^{r} \mathbf{r}-\Omega^{r}(\mathbf{k}) t\right]},
\end{aligned}
$$

where $\Omega=\Omega^{r}+i \Omega^{i}$ and $\mathbf{k}=\mathbf{k}^{r}+i \mathbf{k}^{i}$ are complex to account for both temporal and spatial growth rates. The analysis of $\lambda(\mathbf{r} / t)$ (see the caption of Fig. 1) then gives relations on $\Omega(\mathbf{k})$ that provide the thresholds of convective and absolute instabilities and their corresponding wave vectors. These relations are summarized in Table I, where $\vec{V}_{g}$ is the group velocity of the wave packet and $\left.\overrightarrow{\nabla_{\mathbf{k}^{r}}}\left(\Omega^{i}\right)\right|_{\mathbf{k}_{c}}=\overrightarrow{0}$ means $\left.\frac{\partial \Omega^{i}}{\partial k_{x}^{r}}\right|_{\mathbf{k}_{c}}=0$ and $\left.\frac{\partial \Omega^{i}}{\partial k_{y}^{r}}\right|_{\mathbf{k}_{c}}=0$.

\section{DISPERSION RELATION}

Table I shows that the expression of the dispersion relation $\Omega(\mathbf{k})$ is required to locate the different types of structures that are destabilized at the convective threshold. This 
TABLE I. Relations giving the convective and absolute instability thresholds and their associated wave vectors.

\begin{tabular}{|c|c|c|}
\hline & Convective & Absolute \\
\hline $\begin{array}{l}\text { Unstable } \\
\text { wave vectors }\end{array}$ & $\mathbf{k}^{*}=\mathbf{k}_{c} \in \mathbb{R}$ & $\mathbf{k}^{*}=\mathbf{k}_{a} \in \mathbb{C}$ \\
\hline \multirow{2}{*}{$\begin{array}{l}\text { Wave vectors } \\
\text { obtained from }\end{array}$} & $\left.\overrightarrow{\nabla_{\mathbf{k}^{r}}}\left(\Omega^{i}\right)\right|_{\mathbf{k}_{c}}=\overrightarrow{0}$ & $\left.\overrightarrow{\nabla_{\mathbf{k}^{r}}}\left(\Omega^{i}\right)\right|_{\mathbf{k}_{a}}=\overrightarrow{0}$ \\
\hline & $\left.\overrightarrow{\nabla_{\mathbf{k}^{r}}}\left(\Omega^{r}\right)\right|_{\mathbf{k}_{c}}=\left(\frac{\mathbf{r}}{t}\right)=\overrightarrow{V g}$ & $\left.\vec{\nabla}_{\mathbf{k}^{r}}\left(\Omega^{r}\right)\right|_{\mathbf{k}_{a}}=\overrightarrow{0}$ \\
\hline $\begin{array}{l}\text { Thresholds } \\
\text { obtained from }\end{array}$ & $\Omega^{i}\left(\mathbf{k}_{c}\right)=0$ & $\Omega^{i}\left(\mathbf{k}_{a}\right)=0$ \\
\hline
\end{tabular}

relation is obtained from the model of our setup (Fig. 2) based on the well known feedback optical system first introduced by Akhmanov et al. [15] and later developed by Firth and d'Alessandro [16]. Here, the model is modified to account for the nonlocality and for the noise. It reads

$$
\begin{gathered}
\left(-\nabla_{\perp}^{2}+\frac{\partial}{\partial t}+1\right) n(\mathbf{r}, t)=|F(\mathbf{r})|^{2}+|B(\mathbf{r}, t)|^{2}+\sqrt{\epsilon} \xi(\mathbf{r}, t), \\
B(\mathbf{r}, t)=\sqrt{R} F_{0} e^{i \sigma \nabla_{\perp}^{2}\left[e^{i \chi n(x-h, y, t)} g(\mathbf{r})\right],}
\end{gathered}
$$

where $n(\mathbf{r}, t)$ stands for the refractive index of the nonlinear nematic liquid crystal (LC) layer; $t$ and $\mathbf{r}=(x, y)$ are the time and transverse space variables scaled with respect to the relaxation time $\tau$ and the diffusion length $l_{d} ; R$ is the mirror intensity reflectivity. We have set $\sigma=d / k_{0} l_{d}^{2}$, where $d$ is the slice-mirror distance and $k_{0}$ is the optical wave number of the field. The nonlocality arises from the term $(x-h)$ in the expression of the backward beam $B$, where $h$ represents the lateral shift along the $x$ axis due to the tilted mirror as indicated in Fig. 1. $\xi(\mathbf{r}, t)$ accounts for thermal noise and describes a Gaussian stochastic process of zero mean and correlation $\left\langle\xi^{*}(\mathbf{r}, t) \xi\left(\mathbf{r}^{\prime}, t^{\prime}\right)\right\rangle=\delta\left(\mathbf{r}-\mathbf{r}^{\prime}\right) \delta\left(t-t^{\prime}\right),\left\langle\xi(\mathbf{r}, t) \xi\left(\mathbf{r}^{\prime}, t^{\prime}\right)\right\rangle$ $=0$. The level of noise is controlled by the parameter $\epsilon$. $F$ is the forward input optical field; its transverse profile is accounted for using $F(\mathbf{r})=F_{0} g(\mathbf{r})$, with $g(\mathbf{r})=\exp \left[-\left(x^{2}\right.\right.$ $\left.\left.+y^{2}\right) / w^{2}\right]$ for a Gaussian pump beam of radius $w$ and $g=1$ for the uniform (plane wave) case. $B$ is the backward optical field [16]. The Kerr effect is parametrized by $\chi$, which is

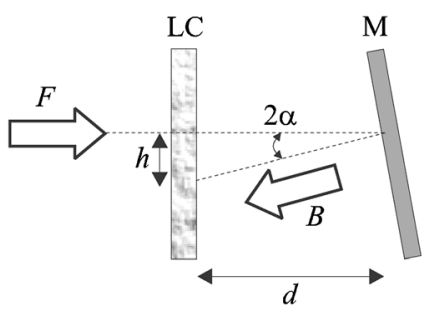

FIG. 2. Schematic sketch of the experimental setup. LC, liquidcrystal layer; $M$, feedback mirror; $F$, input optical field; $B$, backward optical field; $\alpha$, mirror tilt angle; $d$, feedback length. positive (negative) for a focusing (defocusing) medium.

Starting from the above equations, in the plane-wave approximation $[g(\mathbf{r})=1]$ and in the absence of noise $(\epsilon=0)$, a linear stability analysis provides us with the dispersion relation. Assuming perturbations of the stationary state $n_{0}$ $=F_{0}^{2}(1+R)$ in the form $\Delta n(\mathbf{r}, t) \sim \exp i[\mathbf{k} \cdot r-\Omega(\mathbf{k}) t]$ with $\mathbf{k}$ $=\left(k_{x}, k_{y}\right)$, we obtain the following dispersion relation:

$$
\Omega=\Omega^{r}+i \Omega^{i}=-i\left[1+\mathbf{k}^{2}-\mu \sin \left(\sigma \mathbf{k}^{2}\right) \exp \left(i h k_{x}\right)\right],
$$

where $\mu=2 R F_{0}^{2}|\chi|$ is the reduced intensity control parameter. The above expression shows that the presence of $h$ leads to a complex dispersion relation, meaning that, in addition to the classical temporal instabilities ( $\mathbf{k}$ real and $\Omega$ complex), there are also spatial amplifications (k complex).

Our interest now is to determine to what extent the transverse shift influences and more importantly affects the formation of the different types of basic patterns or "modes" that destabilize at convective threshold, their domain of convectivity (up to the absolute threshold), and their properties. Interactions and competitions between such patterns are beyond the scope of the present work.

\section{CONVECTIVE MODES AND THEIR THRESHOLDS}

Conditions defining the convective threshold and the associated wave vectors of a mode for a given value of the shift $h$ are obtained from Table I and Eq. (4). The following set of five equations is then obtained:

$$
\begin{aligned}
& \left.\frac{\partial \Omega^{i}}{\partial k_{x}^{r}}\right|_{\mathbf{k}_{c}}=\mu_{c}\left[2 k_{c_{x}} \sigma \cos \left(\sigma \mathbf{k}_{c}^{2}\right) \cos \left(h k_{c_{x}}\right)\right. \\
& \left.-h \sin \left(\sigma \mathbf{k}_{c}^{2}\right) \sin \left(h k_{c_{x}}\right)\right]-2 k_{c_{x}}=0, \\
& \left.\frac{\partial \Omega^{i}}{\partial k_{y}^{r}}\right|_{\mathbf{k}_{c}}=\mu_{c} 2 k_{c_{y}} \sigma \cos \left(\sigma \mathbf{k}_{c}^{2}\right) \cos \left(h k_{c_{x}}\right)-2 k_{c_{y}}=0, \\
& \left.\frac{\partial \Omega^{r}}{\partial k_{x}^{r}}\right|_{\mathbf{k}_{c}}=-\mu_{c}\left[2 k_{c_{x}} \sigma \cos \left(\sigma \mathbf{k}_{c}^{2}\right) \sin \left(h k_{c_{x}}\right)\right. \\
& \left.+h \sin \left(\sigma \mathbf{k}_{c}^{2}\right) \cos \left(h k_{c_{x}}\right)\right]=V_{g x}, \\
& \left.\frac{\partial \Omega^{i}}{\partial k_{y}^{r}}\right|_{\mathbf{k}_{c}}=-\mu_{c} 2 k_{c_{y}} \sigma \cos \left(\sigma \mathbf{k}_{c}^{2}\right) \sin \left(h k_{c_{x}}\right)=V_{g y}, \\
& \Omega^{i}\left(\mathbf{k}_{c}\right)=-1-\mathbf{k}_{c}^{2}+\mu_{c} \sin \left(\sigma \mathbf{k}_{c}^{2}\right) \cos \left(h k_{c_{x}}\right)=0 .
\end{aligned}
$$

Here, we arbitrarily chose the transverse displacement $h$ along the $x$ axis.

The previous set of Eqs. (5)-(9) leads to two sets of convective solutions defined by

$$
\begin{gathered}
k_{c_{y}}=0, \\
k_{c_{x}}^{(n)}=\frac{n \pi}{h} .
\end{gathered}
$$

We then investigate the family of instabilities for each of these two solutions. Condition (10a) obviously leads to solu- 
tions with no modulation along the $y$ axis since $k_{c_{y}}=0$, and they are called in the following subsection "1D-type convective modes." In contrast, Eq. (10b) yields the full 2D-type convective modes, as we shall see in Sec. III B.

\section{A. The "1D" type convective modes: Vertical rolls}

In the presence of a lateral shift $h$ of the backward beam in the horizontal $x$ direction, the instabilities associated with $k_{c_{y}}=0$ display no modulation along the vertical $y$ axis, i.e., they appear as vertical rolls whose periodicity is fixed by the wave number at threshold $\mathbf{k}_{c}=k_{c_{x}}$. They are similar to those that would be found in a 1D system restricted along the $x$ axis as studied in [1]. Here, we extend the previous study to large values of $h$ and point out that convective pattern formation drastically changes with increasing the value of $h$.

The convective modes are determined in two steps: first, from Eq. (5), we determine the destabilized wave number $k_{c_{x}}(h)$ at threshold. Since periodic functions are present in Eq. (5), there exists an infinite number of solutions for $k_{c_{x}}(h)$ indexed by $[p]$. No analytical expression of $k_{c_{x}}^{[p]}(h)$ exists since Eq. (5) is transcendental. We then determine them numerically. Thus, these wave numbers $k_{c_{x}}^{[p]}(h)$ are injected in Eq. (9) to yield the convective threshold $\mu_{c}^{[p]}(h)$ as

$$
\mu_{c}^{[p]}(h)=\frac{1+k_{c_{x}}^{2[p]}}{\sin \left(\sigma k_{c_{x}}^{2[p]}\right) \cos \left(h k_{c_{x}}^{[p]}\right)} .
$$

Figures 3 and 4 display the evolution of the first six convective thresholds and their associated wave numbers versus $h$ (the $x$ subscript is omitted for the sake of clarity). The convective threshold curves versus $h$ look like tongues and evolve very strongly with $h$. For a given $h$, instabilities with different wave numbers may compete in the regimes where tongues overlap. For each value of $p$, the associated wave number decreases continuously (for $\sigma<0$ ) with $h$. The lowest convective threshold and its associated pattern wave number are determined graphically (bold curves in Figs. 3 and 4). The wave number corresponding to this mode evolves with discontinuity (bold curve of Fig. 4) and the jumps are associated with a change of index $[p]$ of the lowest threshold tongue.

The phase and group velocities $\left(V_{\phi}, V_{g}\right)$ of the mode selected at the lowest convective threshold are plotted in Fig. 5 . We can see that the group velocity is always positive [Fig. 5 (b)] whereas the phase velocity can be either positive or negative, meaning that the rolls can drift upstream [see, e.g., $h=8$ in Fig. 5(a)] or downstream. The change in the sign of the phase velocity occurs at each local minimum of the lowest convective threshold curve (Fig. 5) where the pattern is nondrifting (e.g., $h=4.65$ or 10.55). The minimum of each tongue in Fig. 3 leads to a spatially fixed pattern [null phase velocity in Fig. 5(a)]. This situation corresponds to a lateral shift $h$ multiple of the half-wavelength $\Lambda=2 \pi / k_{c_{x}}^{[p]}$. Physically it means that each time the lateral shift $h$ is such that a fringe (maximum of transverse modulation) of the backward field $B$ comes back in phase or in antiphase with a fringe of the incoming field $F$, the pattern does not drift and its thresh-
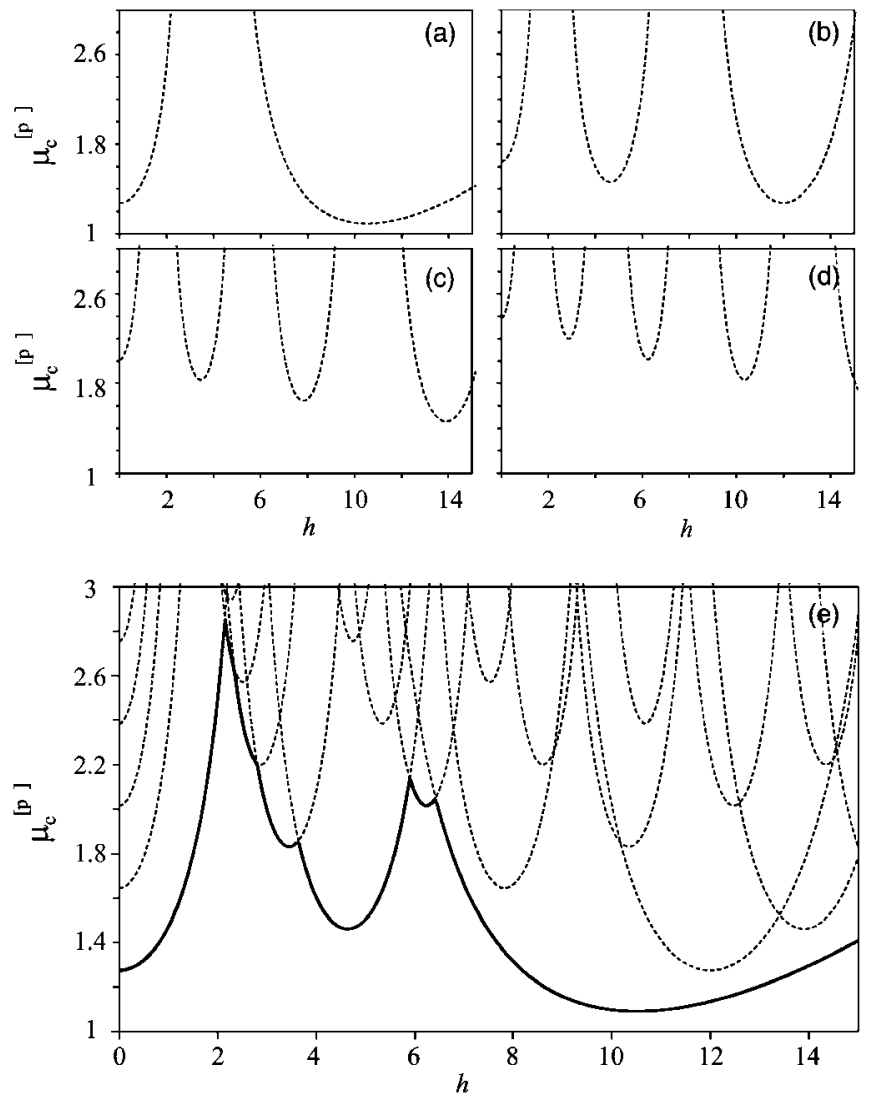

FIG. 3. Evolutions of the convective thresholds of vertical rolls vs $h$ for (a) $p=1$, (b) $p=2$, (c) $p=3$, and (d) $p=4 . \sigma=-17$ and $\chi$ $=1$. (e) Evolution of the primary threshold vs $h$ (bold curve).

old is minimum since the nonlinear Kerr effect is optimum.

\section{B. The "2D" type convective modes: Horizontal rolls and rectangular lattices}

These modes are specific to the full 2D dynamics and are associated with the second condition [Eq. (10b)], namely $k_{c_{x}}^{(n)}=\frac{n \pi}{h}$. As done previously, we determine the variation of the wave vectors and thresholds versus $h$ by combining the

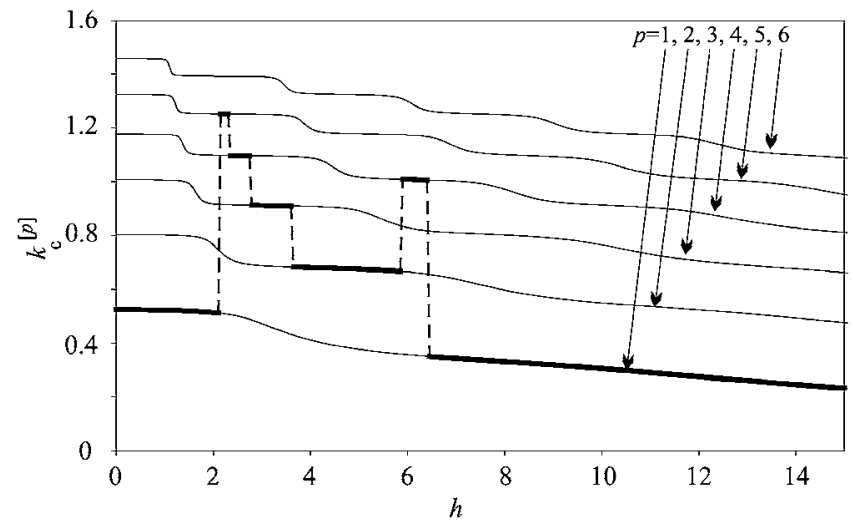

FIG. 4. Evolution of the first six convective wave numbers vs $h$ corresponding to Fig. 3. The bold curve follows the wave number selected at the primary convective threshold. 


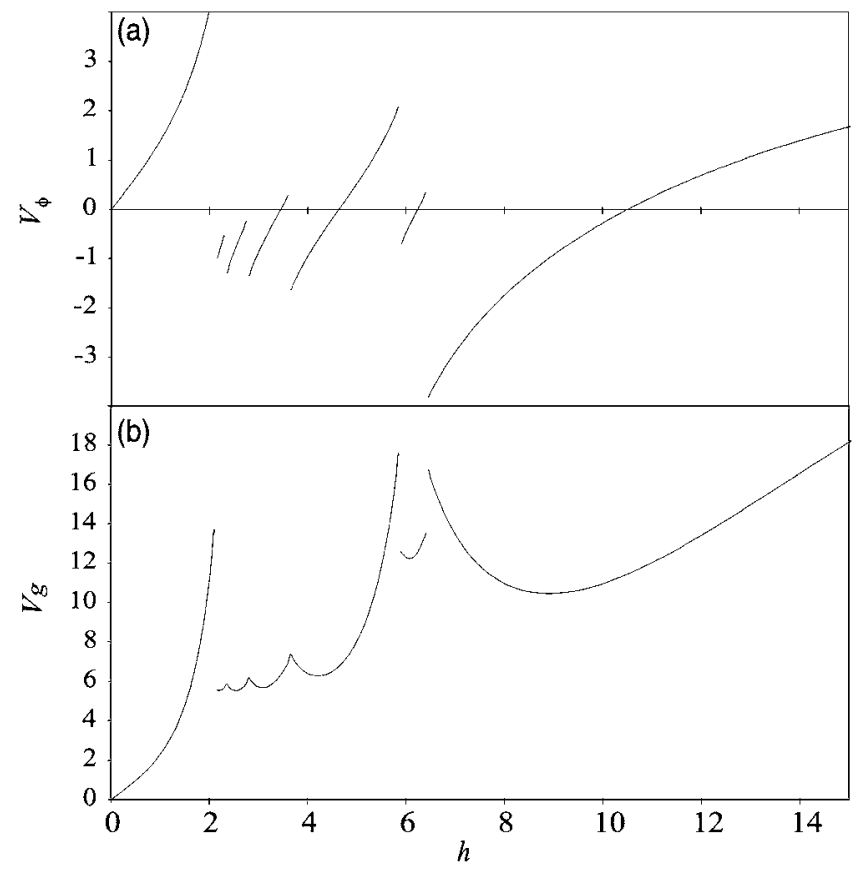

FIG. 5. Evolution of the phase and group velocities $\left(V_{\phi}\right.$ and $V_{g}$, respectively) for the mode of lowest threshold vs $h$ corresponding to Fig. 3.

set of Eqs. (5)-(9) with the condition on $k_{c_{x}}^{(n)}$. Inserting this condition in Eq. (9) leads to a series of thresholds for convective instabilities,

$$
\mu_{c}^{(n)}(h)=(-1)^{n} \frac{1+k_{c}^{2_{(n)}}}{\sin \left(\sigma k_{c}^{2_{(n)}}\right)}, \quad n \in \mathbb{N}
$$

with

$$
k_{c}^{2(n)}=k_{c_{x}}^{2(n)}+k_{c_{y}}^{2(n)}=\left(\frac{n \pi}{h}\right)^{2}+k_{c_{y}}^{2(n)} .
$$

The striking feature of Eq. (12) is that the threshold is independent of $h$, and is equal to its value in the absence of drift $(h=0)$, except for the term $(-1)^{n}$. So, their wave number and threshold are constant with $h$. As a consequence, regardless of $h$, the convective thresholds coincide with those of the system without drift depending on the parity of $n$,

$$
\begin{aligned}
& \mu_{c}^{(n)}(h, \sigma)=\mu_{c}^{(n)}(0, \sigma) \text { for } n \text { even, } \\
& \mu_{c}^{(n)}(h, \sigma)=\mu_{c}^{(n)}(0,-\sigma) \text { for } n \text { odd } .
\end{aligned}
$$

For negative (positive) values of $\sigma$, the convective thresholds are those of the negative (positive) nonlinearity system without drift for even $n$, and those of the positive (negative) nonlinearity for odd $n$.

The specific values of the wave numbers $k_{c}^{(n)}$ are obtained for the minimum of $\mu_{c}^{(n)}(h)$ versus $k_{c}^{(n)}$ in Eq. (12) that gives

$$
\tan \left(\sigma k_{c}^{2_{(n)}}\right)=(-1)^{n} \sigma\left(1+k_{c}^{2_{(n)}}\right) .
$$

This trigonometric equation admits, for a fixed value of $n$, multiple wave-number solutions indexed by $p$, as in Sec.
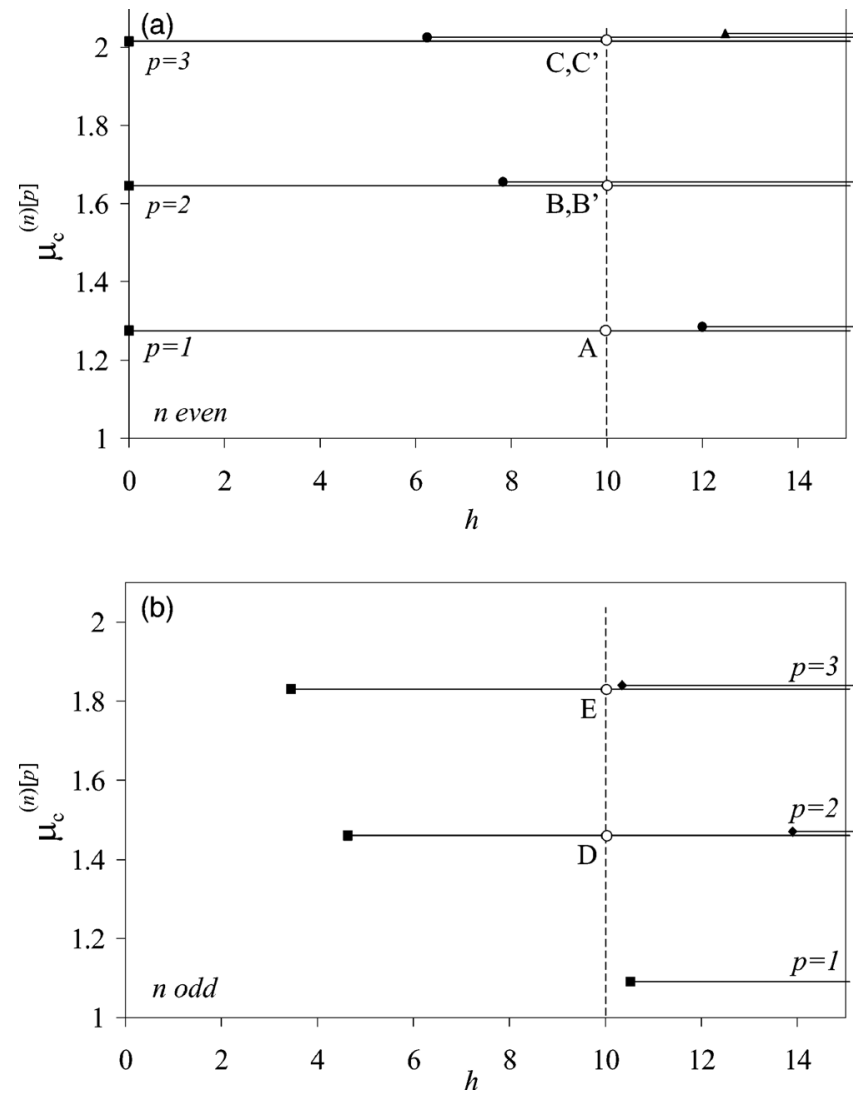

FIG. 6. Evolutions of the 2D type convective mode thresholds $\mu_{c}^{(n)[p]}$ vs $h$ for three first values of $p$. (a) Even values of $n(n=0$ squares, $n=2$ diamonds, $n=4$ triangles) and (b) odd values of $n$ ( $n=1$ squares, $n=3$ diamond). Note that for a given value of $n$, the threshold value increases with the $p$ index. The domains of existence of the modes $k_{c}^{(n)[p]}$ are located to the right of the squares, diamonds, and triangles ending the half-lines. Letters $A-E$ correspond to threshold values of the patterns of Fig. 8. Threshold curves (that are degenerate) have been slightly shifted for the sake of visibility. $\sigma=-17, \chi=1$.

III A. To summarize, convective solutions are characterized by two indices $(n)[p]$. Each $(n)[p]$ mode with a wave number $k_{c}^{(n)[p]}$ and associated threshold $\mu_{c}^{(n)[p]}$ exists within a bounded domain set by equality (13) since $k_{c_{y}}^{(n)[p]}$ exists only if

$$
h \geqslant h_{c}^{(n)[p]}=\frac{n \pi}{k_{c}^{(n)[p]}} .
$$

These two properties are illustrated in Fig. 6, where the evolution of the convective thresholds for the lower indices $(n$ $\leqslant 4 ; p \leqslant 2$ ) is plotted versus the lateral shift $h$. As explained above, these thresholds are completely independent of the shift $h$ and the existence condition selects the domains of the threshold curves that reduce to half-lines starting at $h_{c}^{(n)[p]}$. The transverse displacement does not change here the threshold value nor the mode composition of convective modes, contrary to the $1 \mathrm{D}$ type modes. Only the nature of the pattern emerging at the lowest threshold depends on $h$. 

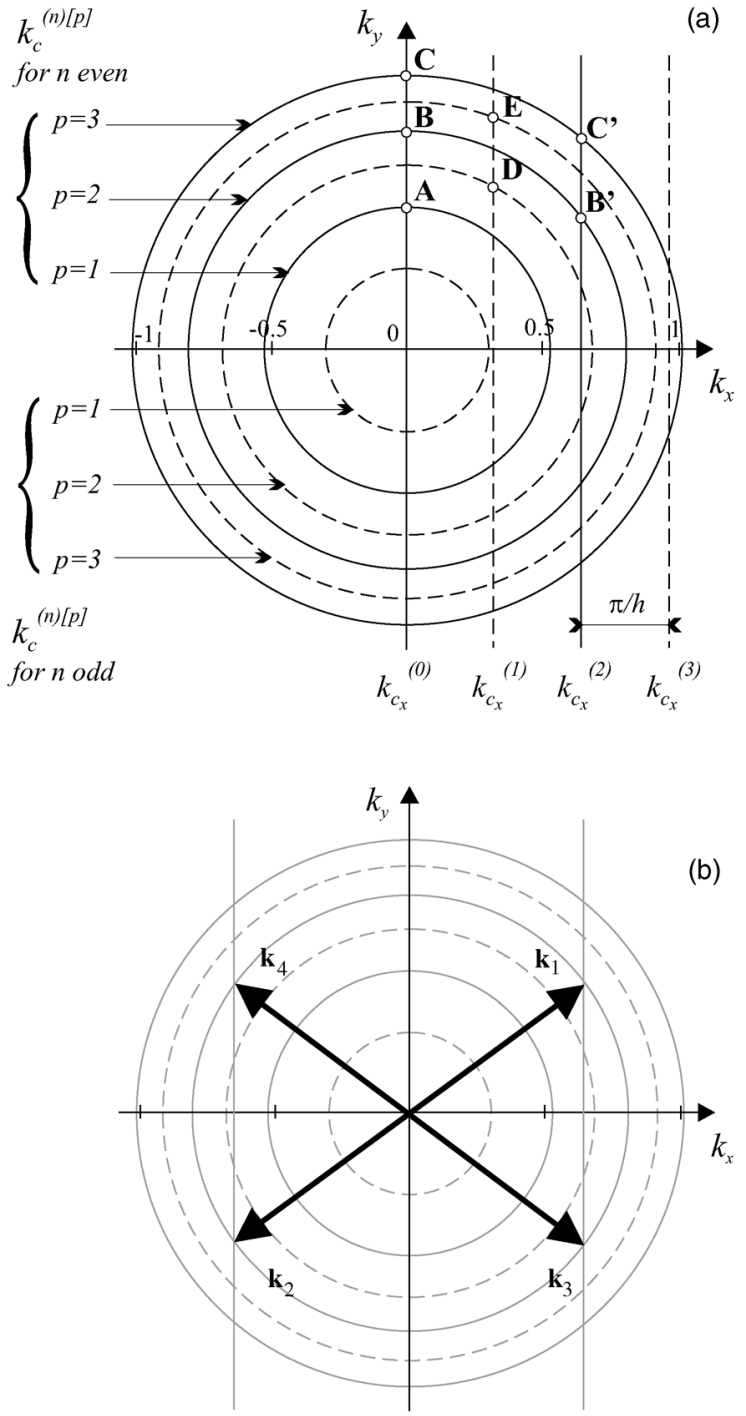

FIG. 7. (a) 2D type convective mode wave-vector locations depending on their indexes $(n)$ and $[p]$. The possible wave-vector ends are found at the crossings of a circle of radius $k_{c}^{(n)[p]}$ and a vertical line defined by $k_{c_{x}}^{(n)}=\frac{n \pi}{h}$ of the same draw type (continuous or dashed). Only the wave-vector ends belonging to the first quadrant are represented. Dots labeled $A, B, C, D, E, B^{\prime}, C^{\prime}$ correspond to modes (0)[1], (0)[2], (0)[3], (1)[2], (1)[3], (2)[2], (2)[3]. (b) Full composition of the four wave vectors $\mathbf{k}_{1}$ to $\mathbf{k}_{4}$ contributing to mode $B^{\prime}$. Corresponding near- and far-field transverse modes are depicted in Fig. 8 and their thresholds in Fig. 6.

The wave-vector construction of these 2D convective modes is the following. For a given $(n)[p]$ mode, $(n)$ gives the wave-vector $x$ component defined by $k_{c_{x}}^{(n)}=\frac{n \pi}{h}$ and $[p]$ gives the modulus $k_{c}^{(n)[p]}$ of the wave vectors composing the mode. Thus, the location of the end of the wave vectors composing a convective mode $(n)[p]$ is defined by the intersection of a circle of radius $k_{c}^{(n)[p]}$ and a vertical line defined by $k_{c_{x}}^{(n)}=\frac{n \pi}{h}$ [Fig. 7(a)]. An example of wave-vector composition for the convective mode $(n)[p]=(2)[2]$ is given in Fig. 7(b). The given wave-vector location description gives the two wave vectors $\mathbf{k}_{1}$ (and $\mathbf{k}_{3}$ ). The $\mathbf{k}_{1}$ wave-vector end corresponds to the dot $B^{\prime}$ in Fig. 7(a). Their two opposite wave

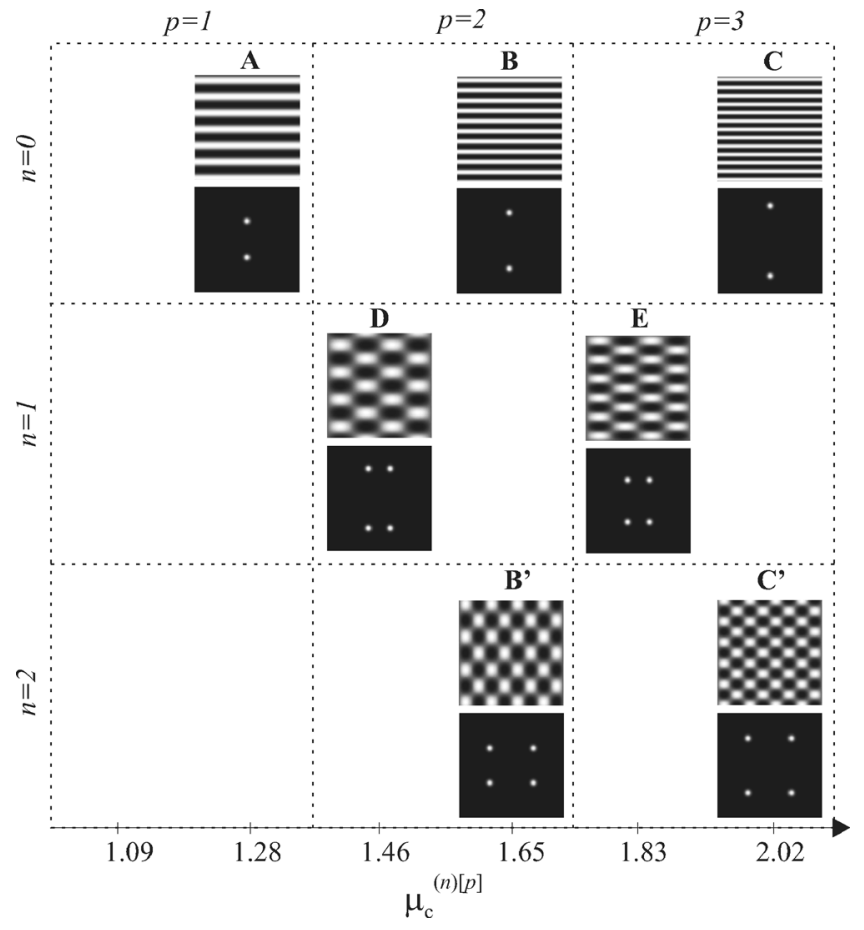

FIG. 8. Convective 2D modes corresponding to the $A-E$ wave vectors presented in Fig. 7. Their convective thresholds are given by the dots in Fig. 6. Top pictures: near field, bottom: far field.

vectors $\mathbf{k}_{2}=-\mathbf{k}_{1}$ and $\mathbf{k}_{4}=-\mathbf{k}_{3}$ also enter the mode composition since Eq. (14) involves the square of $k_{c}^{(n)}$. So such a convective mode is likely to produce a rectangular pattern because this yields a weaker symmetry breaking than rolls.

Globally, two types of 2D convective modes are obtained: horizontal rolls $(n=0)$ and rectangular lattices $(n \neq 0)$, as illustrated in Fig. 8. Examples of convective modes corresponding to the wave-vector constructions presented in Fig. 7(a) are plotted in Fig. 8 for $h=10$ in the conditions of Fig. 6. Depending on the pump parameter $\mu$, horizontal rolls and rectangular lattices are obtained with different wave-number values and wave-vector compositions. Threshold degeneracy is observed for, e.g., the horizontal roll mode $B$ and the rectangular lattice mode $B^{\prime}$ leading to mode competition at the onset of their appearance.

Finally, inspecting the real part of $\Omega(k)$,

$$
\Omega^{r}\left(\mathbf{k}_{c}\right)=\mu_{c} \sin \left(\sigma \mathbf{k}_{\mathbf{c}}{ }^{2}\right) \sin \left(h k_{c_{x}}\right),
$$

one can see that the above expression [Eq. (16)] vanishes regardless of $h$ for the 2D type modes [Eq. (10b)]. This implies that the phase velocity of these modes at convective threshold, defined by $v_{\phi}=\frac{\Omega^{r}\left(\mathbf{k}_{c}\right)}{\mathbf{k}_{c}}$, is identically zero. In other words, all the 2D type modes are nondrifting at convective threshold despite the presence of the drift $(h \neq 0)$. This striking feature has also been predicted in other fields of nonlinear sciences [17] and seems not to be specific to our system but rather to be generic.

\section{Numerical simulations}

So far, all the convective modes have been obtained for an ideal configuration where noise is neglected and the incident 
wave is a plane wave. However, our experimental system is composed of a liquid crystal layer that is noisy and pumped by a Gaussian beam. The changes induced by these two parameters in the numerical simulations as compared to the analytical predictions at the primary convective threshold are the following. Concerning the noise, as mentioned in the Introduction, when noise is absent $(\epsilon=0)$, any initial perturbation is amplified but advected away such that no pattern is observed at long times. On the other hand, when noise is present, a permanent pattern is observed resulting from the continuous spatial perturbation source. The effect of noise is thus to sustain the structures in the regime of convective instability. The level of noise used here, $\epsilon=0.01$, comes from a previous quantitative determination carried out in our experimental setup [18]. Taking into account the Gaussian profile for the input beam in the numerical simulations does not change the global scenario of the convective mode appearance at the primary threshold versus $h$ nor their wave-vector composition. Only the numerical convective threshold values are shifted up (as compared to the analytical ones) due to the finite width of the Gaussian beam. These shifts decrease with the transverse aspect ratio $\eta$ (beam width $2 w$ divided by the pattern wavelength $\Lambda_{\infty}$ calculated in the plane-wave case). In the conditions of our experiments, this ratio is around 30 ( $2 w \approx 3600 \mu \mathrm{m}$ and $\Lambda_{\infty} \approx 120 \mu \mathrm{m}$ ) yielding e.g., at $h=0$, the convective threshold value $\mu_{c}=1.52$ for the experimental conditions while the predicted one is $\mu_{c}=1.28$ for the ideal system. Thus, the numerical simulations carried out for realistic experimental conditions, i.e., including a Gaussian incident beam and a noise source term, allow us to predict the parameter domain in which NSS should be observed experimentally together with their wave-vector content and their thresholds.

An example of the structures that will destabilize at the primary convective threshold for different values of the lateral shift parameter $h$ is given in Fig. 9 for a negative nonlinearity $(\sigma)$ and with both noise and Gaussian input beam. As $h$ increases from 0, horizontal rolls $\left(k_{c_{x}}=0\right)$ appear first up to $h \approx 8.2$, then vertical rolls $\left(k_{c_{y}}=0\right)$ for $8.2<h<10.5$, and rectangular lattices when $h>10.5\left(k_{c_{x}}^{(n)}=\frac{n \pi}{h}\right.$ with $\left.n>0\right)$. The threshold values can be lower than those observed for the system without transverse displacement, as was done experimentally in Ref. [19] ( $h>8$ in Fig. 9). The sequence of observed noise-sustained modes strongly depends on the sign of $\sigma$, since for positive values of $\sigma$ (not represented here) only horizontal rolls and rectangular lattices are observed at the primary convective threshold. We do not discuss here patterns resulting from the combination of convective modes (e.g., at $h \approx 8$, where the horizontal and vertical roll modes have the same convective threshold).

As we can remark from Fig. 9, the NSS mimic the convective modes obtained previously for an ideal system.

\section{ABSOLUTE THRESHOLDS OF THE CONVECTIVE MODES}

Convective modes can be observed as long as the threshold for absolute instabilities $\mu_{a}$ is not reached. If $\mu \geqslant \mu_{a}$, the

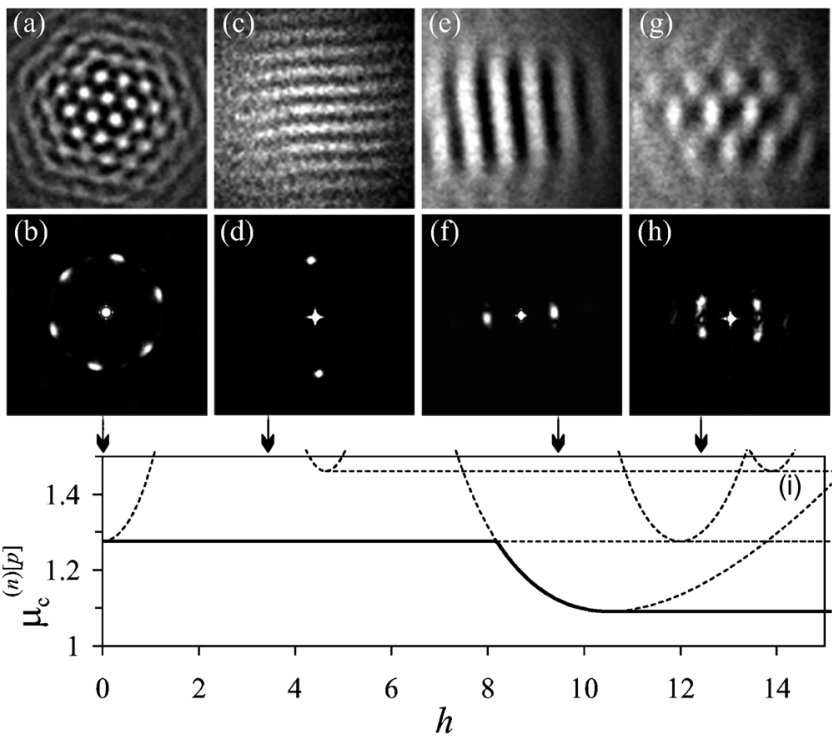

FIG. 9. (a)-(h) Noise-sustained patterns at the primary threshold obtained from numerical simulations carried out for the experimental conditions. The snapshots of the patterns are represented for near (top) and far (bottom) field, respectively. The numerical simulation parameters are (a), (b) $h=0$ and $\mu=1.52$, (c), (d) $h=3.5$ and $\mu$ $=1.62$, (e), (f) $h=9.7$ and $\mu=1.62$, (g), (h) $h=12.5$ and $\mu=1.62, \chi$ $=1, \sigma=-17, \omega=180 l_{d}$, and $\epsilon=0.01$. (i) Synthesis of convective threshold curves of $1 \mathrm{D}$ and 2D type convective modes vs $h$. The sizes of the near- and far-field pictures are, respectively, 130 $\times 130 l_{d}^{2}$ and $1.77 \times 1.77 l_{d}^{-2}$.

initial perturbations of the homogeneous state are amplified all over the space. To know the extension of the convective and absolute domains of the previous convective modes, we need to determine their absolute thresholds. As has been detailed in Sec. I, it is necessary to consider complex wave vectors to characterize the spreading mechanism of the wave packet resulting from an initial local perturbation. Namely, a complex wave vector $\mathbf{k}$ is defined by (for clarity)

$$
\mathbf{k}=k_{x} \cdot \vec{e}_{x}+k_{y} \cdot \vec{e}_{y}=\left(k_{x}^{r}+i k_{x}^{i}\right) \cdot \vec{e}_{x}+\left(k_{y}^{r}+i k_{y}^{i}\right) \cdot \vec{e}_{y},
$$

where $\left(\vec{e}_{x} ; \vec{e}_{y}\right)$ are the Cartesian orthogonal unitary vectors of the transverse plane, and $\left(k_{x} ; k_{y}\right)$ and the $x$ and $y$ components of $\mathbf{k}$. Then, $\mathbf{k}^{2}$ reads

$\mathbf{k}^{2}=k_{x}^{2}+k_{y}^{2}=(\underbrace{k_{x}^{r^{2}}+k_{y}^{r^{2}}-k_{x}^{i^{2}}-k_{y}^{i^{2}}}_{A})+\underbrace{22\left(k_{x}^{r} k_{x}^{i}+k_{y}^{r} k_{y}^{i}\right.}_{B})=A+i B$.

We now plug Eqs. (17) and (18) into the dispersion relation [Eq. (4)]. After lengthy but straightforward calculations, we obtain

$$
\begin{aligned}
\Omega^{r}= & B-\mu \exp \left(-k_{x}^{i} h\right)\left[\cos (\sigma A) \sinh (\sigma B) \cos \left(k_{x}^{r} h\right)\right. \\
& \left.+\sin (\sigma A) \cosh (\sigma B) \sin \left(k_{x}^{r} h\right)\right], \\
\Omega^{i}= & -1-A+\exp \left(-k_{x}^{i} h\right)\left[\sin (\sigma A) \cosh (\sigma B) \cos \left(k_{x}^{r} h\right)\right. \\
& \left.-\cos (\sigma A) \sinh (\sigma B) \sin \left(k_{x}^{r} h\right)\right] .
\end{aligned}
$$

The conditions of Table I expressed in terms of the complex 
components lead to the following five relations:

$$
\begin{aligned}
& \left.\frac{\partial \Omega^{r}}{\partial k_{y}^{r}}\right|_{\mathbf{k}_{a}}=0, \\
& \left.\frac{\partial \Omega^{i}}{\partial k_{y}^{r}}\right|_{\mathbf{k}_{a}}=0, \\
& \left.\frac{\partial \Omega^{r}}{\partial k_{x}^{r}}\right|_{\mathbf{k}_{a}}=0, \\
& \left.\frac{\partial \Omega^{i}}{\partial k_{x}^{r}}\right|_{\mathbf{k}_{a}}=0, \\
& \Omega^{i}\left(\mathbf{k}_{a}\right)=0 .
\end{aligned}
$$

These relations determine both the values of the absolute threshold and of the wave-vector components, i.e., $\mu_{a}, k_{x_{a}}^{r}$, $k_{x_{a}}^{i}, k_{y_{a}}^{r}$, and $k_{y_{a}}^{i}$, respectively. We now proceed to the analysis of the two types of instabilities obtained in the preceding section, namely the " $1 \mathrm{D}$ " and " $2 \mathrm{D}$ " convective modes.

\section{A. "1D" type modes (vertical rolls)}

In the case of vertical rolls, the system of the five previous equations [Eqs. (20a)-(20e)] reduces to three [Eqs. (20c)-(20e)] since there is no component along the $y$ axis (see Sec. III A). No exact analytical expressions of $\mu_{a}, k_{x_{a}}^{r}$, and $k_{x_{a}}^{i}$ have been derived from this set of equations. However, approximated analytical expressions of the latter variables can be obtained from a Taylor expansion of the dispersion relation [Eq. (4)] following the same approach as in $[1,20]$. Here we include the second-order cross-coupling term $1 / 2\left(\partial^{2} \Omega / \partial \mathbf{k} \partial \mu\right)\left(\mathbf{k}-\mathbf{k}_{c}\right)\left(\mu-\mu_{c}\right)$, which was not taken into account in previous works $[1,20]$. In these conditions, the approximated analytical values and numerically solved exact values of the absolute threshold agree very well even for nonsmall values of $\left(\mathbf{k}-\mathbf{k}_{c}\right)$ or $\left(\mu-\mu_{c}\right)$ (Fig. 10). As can be seen from Fig. 10, $\left(\mu-\mu_{c}\right)$ is around 0.4 for $h \approx 8$, which is beyond the conditions for a Taylor expansion, but taking into account the cross-coupling term gives an excellent evaluation of the expected absolute threshold value (black solid line of Fig. 10) even in these conditions. Indeed, without the presence of the second-order cross-coupling term, the approximated value of the absolute threshold would be $20 \%$ far from the alternative value leading to large errors for the width of the purely convective regimes. Finally, the system [Eqs. (20c)-(20e)] is numerically solved to get exact values of $\mu_{a}, k_{x_{a}}^{r}$, and $k_{x_{a}}^{i}$. One has then to ensure that the obtained values actually correspond to a saddle point $[3,21]$ by checking first the pinching condition as shown in Fig. 12. From the example of Fig. 11, at saddle point, the pinching condition of the curve $\Omega^{i}=0$ in the complex plane defined by $\left(k^{r}, k^{i}\right)[\mathrm{Fig}$. $12(\mathrm{c})]$ is satisfied. Secondly, one has to check that this saddle point corresponds to the one associated with the absolute threshold. Indeed, Papoff and Zambrini [22] mention that

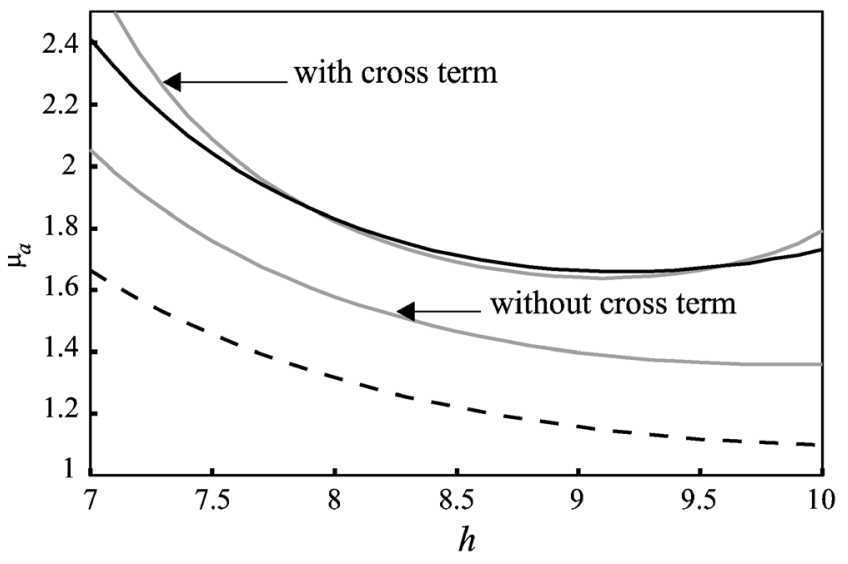

FIG. 10. Influence of the second-order cross term in the Taylor expansion of the dispersion relation for nonsmall values of $(\mu$ $-\mu_{c}$ ). Numerically solved exact convective (dashed line) and absolute (black solid line) thresholds for $\sigma=-17$. In gray, the approximate values of the absolute threshold with and without a secondorder cross term are shown.

due to the exponential term in the dispersion relation, there exists a countable infinity of saddle points. To avoid the nondesired saddle points, we follow by continuity from the situation $h=0$ the one actually corresponding to the absolute threshold up to the desired $h$ value.

The absolute thresholds obtained in the conditions of Fig. 3 are plotted in Fig. 13. Note, for mode $[p]=1$ around $h=9$, the relatively wide regions of the convective regime (gray region between dashed and solid lines). We emphasize here that for $h=8$, the mode that destabilizes first does not become the first absolutely unstable mode. Moreover, by varying the control parameter $\mu$ and following the spatiotemporal evolution of a given convective mode, we have observed that its phase velocity $v_{\phi}$ at absolute threshold generally differs from that at convective threshold. The resulting striking feature is that a nondrifting mode at convective threshold will be traveling at the absolute one and vice versa.

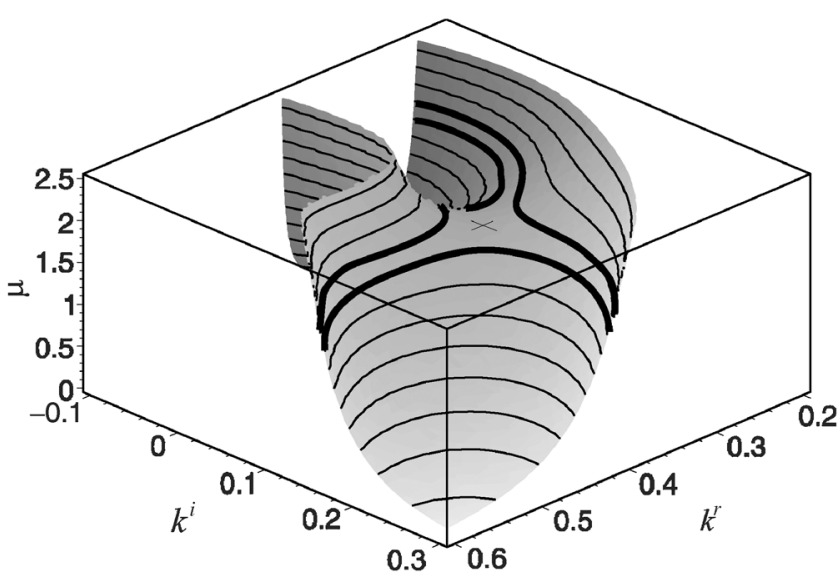

FIG. 11. $\Omega^{i}=0$ surface in $\left(k^{r}, k^{i}, \mu\right)$ space showing the saddle point around the absolute threshold point $\mu_{a}=1.8$ (cross). Bold level lines correspond to $\mu=1.6$ (below absolute threshold) and $\mu$ $=1.8$ (above absolute threshold). $\sigma=-17$ and $h=10$. 


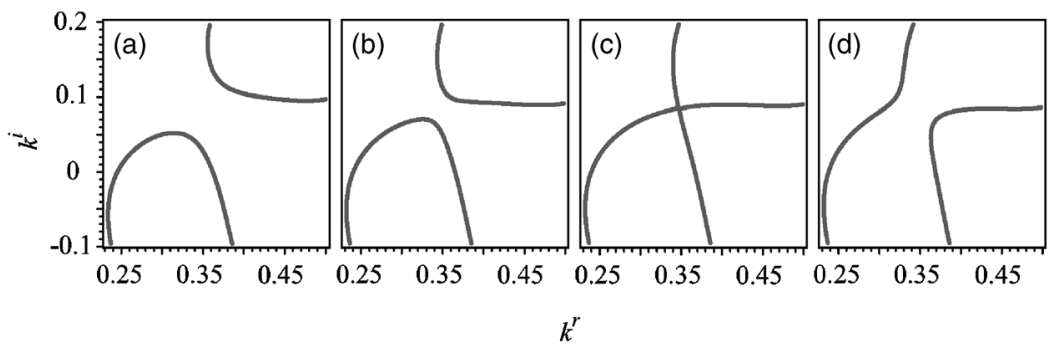

FIG. 12. Pinching condition observed for $\Omega^{i}$ $=0$ when crossing the absolute threshold at $\mu$ $=1.731$. (a) $\mu=1.6$, (b) $\mu=1.7$, (c) $\mu=1.731$, and (d) $\mu=1.8$. Same parameters as Fig. 11 .

\section{B. "2D" type modes (horizontal rolls and rectangular lattices)}

The situation of 2D type modes is complicated because a system of five equations should be solved. However, two observations lead to a significant reduction of the degree of the system complexity. The first one is that at absolute threshold

$$
k_{y_{a}}^{i}=0
$$

Indeed, there is no transverse displacement along the $y$ axis, hence there is no spatial amplification along this axis at absolute threshold. This situation is common to other systems experiencing drift effects as, in particular, for optical parametric oscillators in the presence of walk off [23]. The second one that is valid only for the horizontal rolls,

$$
k_{x_{a}}^{r}=0
$$

originates, in the absence of modulation along the $x$ axis, from these modes.

Starting from these properties, we first develop an analytical expression of the absolute threshold for the horizontal rolls. Putting Eqs. (21) and (22) in Eqs. (20a)-(20e), we straightforwardly obtain $B=0$ leading to the following system $\left(k_{y_{a}}^{r} \neq 0\right)$ :

$$
\begin{gathered}
\mu_{a} \exp \left(-k_{x_{a}}^{i} h\right) \sin (\sigma A)=1+A, \\
\mu_{a} \exp \left(-k_{x_{a}}^{i} h\right) \sigma \cos (\sigma A)=1,
\end{gathered}
$$

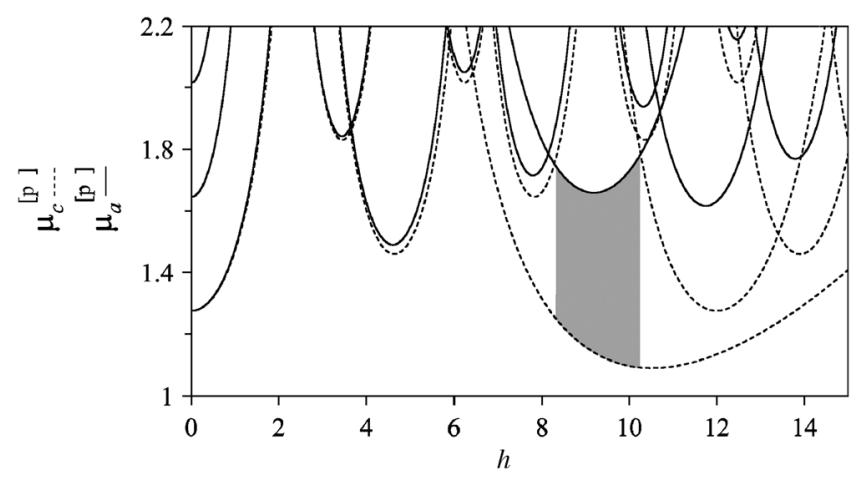

FIG. 13. Evolution of the six first convective $\mu_{c}$ (dashed lines) and absolute $\mu_{a}$ (plain lines) thresholds of vertical rolls vs $h$ for $\sigma=-17, \chi=1$. The gray region corresponds to the width of the convective regime of mode $[p]=1$ for $h \approx 9$.

$$
\mu_{a} \exp \left(-k_{x_{a}}^{i} h\right)\left[2 \sigma \cos (\sigma A) k_{x_{a}}^{i}-h \sin (\sigma A)\right]=2 k_{x_{a}}^{i} .
$$

Substituting Eqs. (23a) and (23b) into Eq. (23c) gives

$$
h(1+A)=0 .
$$

So, either $h=0$ or $A=-1$. Since here $h \neq 0$, then $A=-1$ leading to a nonphysical solution. Thus, no absolute threshold is found for the horizontal rolls. The analytical development of the absolute threshold carried out for the rectangular lattices also leads to a system that does not possess absolute thresholds. We have numerically solved the system of Eqs. (20a)-(20e) together with the approximated expansion of the dispersion relation, and no saddle point was found for the $2 \mathrm{D}$ type modes. This is in agreement with the numerical simulations performed with the parameter set of Fig. 3 with $h=2$, where only convective horizontal rolls have been found up to $2 \mu_{c}(\mu \in[1.3 ; 2.8]$, i.e., in a region where only horizontal rolls are predicted). From these investigations, one may conjecture that the $2 D$ type modes are purely convective.

\section{EXPERIMENTAL RESULTS}

The main goal of this section is to compare the experimental patterns observed at the primary threshold with the analytical predictions and the numerical simulations carried out in realistic conditions. The comparison is mostly performed on the wave-vector composition of the convective modes together with the evolution of their threshold $\mu_{c}(h)$ and wave number $k_{c}$ with the lateral shift $h$.

The experiments are performed on the setup already described in $[18,24]$ with the main difference of having a feedback mirror tilted by an angle $\alpha$ (see Fig. 2). A 50- $\mu$ m-thick layer of $E_{7}$ homeotropically aligned nematic liquid crystal at $22{ }^{\circ} \mathrm{C}$ is irradiated by a $532 \mathrm{~nm}$ frequency doubled $\mathrm{Nd}: \mathrm{YVO}_{4}$ laser with a beam radius of $1.8 \mathrm{~mm}$. The response time $\tau$ and diffusion length $l_{d}$ are $2.3 \mathrm{~s}$ and $10 \mu \mathrm{m}$, respectively [25]. Thus, for a typical feedback length $d=-2 \mathrm{~cm}$, the angle $\alpha$ is of order of $2.5 \mathrm{mrad}$ for $h \approx 10 l_{d}=100 \mu \mathrm{m}$ (to be compared with the pattern wavelength in the conditions of a uniform pump profile $\Lambda_{\infty}=120 \mu \mathrm{m}$ ). In the following, $h$ will be given in units of $l_{d}$ to keep the same units as for analytical predictions.

Starting from the "homogeneous" state (i.e., without any transverse modulation except for the Gaussian profile) and increasing the transverse shift $h$, we successively observe, at the first convective instability threshold, noise-sustained horizontal rolls [Figs. 14(c) and 14(d)], vertical rolls [Figs. 14(e) and 14(f)], and rectangular lattices [Figs. 14(g) and 


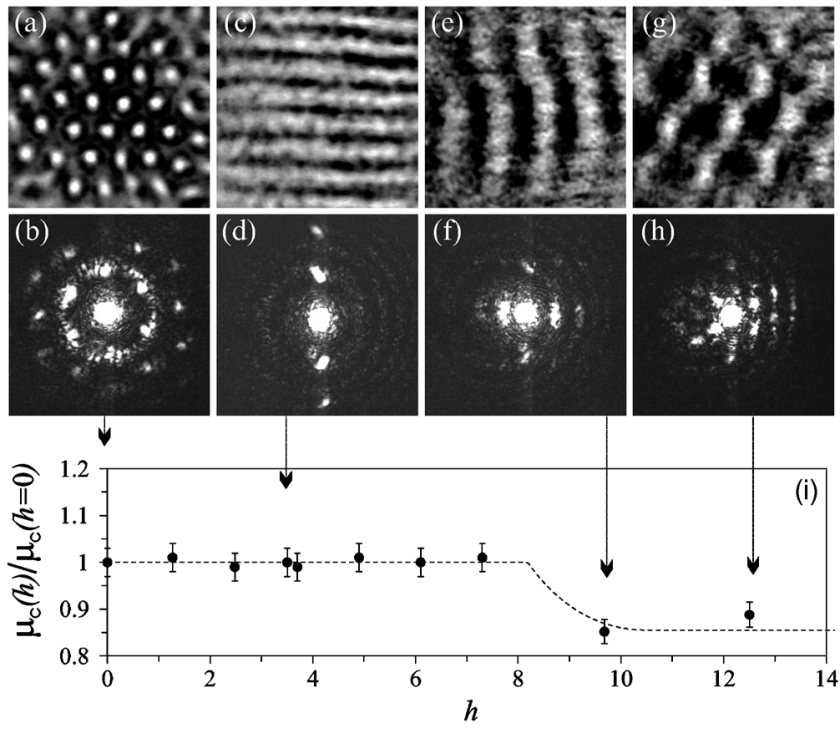

FIG. 14. Near-field (upper row) and far-field (lower row) 2D experimental noise-sustained patterns observed for different values of the shift $h$ (in units of $l_{d}$ ) at the first convective instability threshold. $\sigma=-17, d=-20 \mathrm{~mm}$. $\mu_{c}$ is measured in units of the hexagon threshold intensity at $h=0$ evaluated with $10 \%$ accuracy. $h=0$ hexagons (a), (b), $h=3.5$ horizontal rolls (c), (d), $h=9.7$ vertical rolls (e), (f), and $h=12.5$ rectangular lattices (g), (h) are observed. (i) Experimental values of the normalized convective threshold $\mu_{c}(h) / \mu_{c}(h$ $=0)$ (dots). The dotted line corresponds to its analytical value [Fig. $9(i)]$. The size of pictures is $1.3 \times 1.3 \mathrm{~mm}^{2}\left(130 \times 130 l_{d}^{2}\right)$ for the near fields and $15 \times 15 \mathrm{mrad}\left(1.77 \times \times 1.77 l_{d}^{-2}\right)$ for the far fields.

14(h)]. This scenario exactly follows the one predicted by our analytical study illustrated in Fig. 9. Indeed, starting from the situation in which the transverse shift $h$ is absent, so that the hexagon [Figs. 14(a) and 14(b)] is the only possible absolute pattern, we increase from zero the value of $h$ and successively recover the three convective modes obtained by numerical simulations and predicted by our theoretical analysis.

Several qualitative properties of these structures have been checked.

(i) The convective threshold of the horizontal rolls does not evolve with $h$, as can be seen in Fig. 14 for $0<h<8$. This result was predicted in Sec. III B and plotted in Fig. 6. It reveals that the convective threshold of horizontal rolls is the same as that of the hexagonal pattern. We have also checked that the convective threshold of rectangular lattices remains constant with $h$ for $h>10.5$.

(ii) The wave number at convective threshold of horizontal rolls or rectangular lattices does not change with $h$ for constant input intensity parameter (Fig. 15). This property is a direct consequence of the previous property of independence of the convective threshold upon $h$ [Eq. (12)].

(iii) The rectangular lattices are not drifting at threshold. Indeed, as we have checked in Fig. 16(c), the profile of its cross section does not propagate along the transverse shift direction, meaning that its phase velocity vanishes. This is also the case for horizontal rolls. This property is remarkable since it is nonintuitive and in contrast with the commonly widespread situation where convection leads to propagating

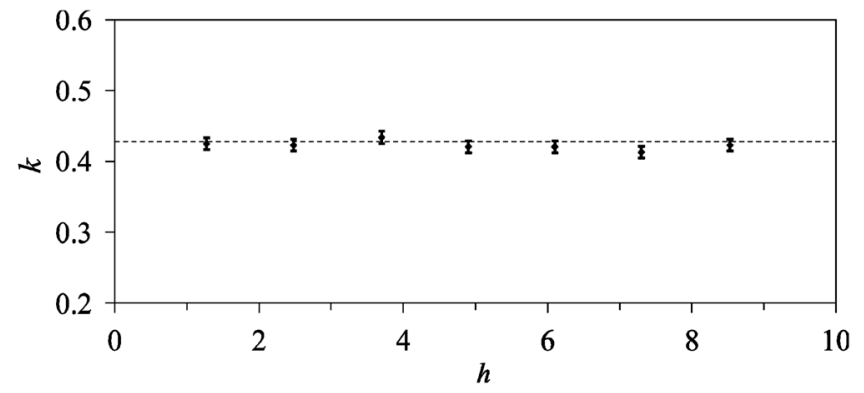

FIG. 15. Evolution of the wave number (in units of $l_{d}^{-1}$ ) of the horizontal roll mode (0)[1] vs $h$ at convective threshold.

patterns. Let us mention that there exists a temporal dynamics of the noise-sustained rectangular lattices at convective threshold. It looks like alternation between the two rolls composing the pattern.

(iv) The vertical rolls are drifting patterns and have a threshold value lower than that of the hexagonal structure in the absence of transverse shift (see region $8<h<10.5$ in Fig. 14).

We have also performed a quantitative comparison of the predictions of the model with the experimental observations in the case of the vertical rolls. Since these patterns have the same threshold and wave number as those of an equivalent 1D system, we have realized such a system by means of cylindrical lenses [24], thus keeping only the wave-vector component along the transverse shift axis $\left(k_{x}\right)$. The results are plotted in Fig. 17. The determination of the convective threshold is carried out by using the instability convective signature based on the evolution of pattern region upstream

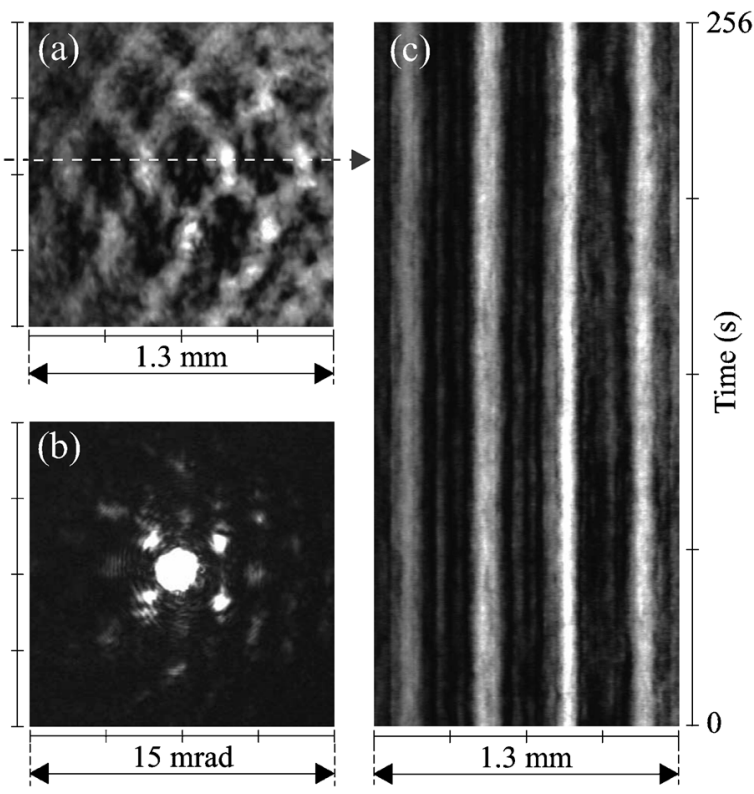

FIG. 16. (a) Experimental nondrifting noise-sustained rectangular lattice for a non-null lateral shift $h$ at first convective threshold. Upper, near field; lower, far field. (b) Temporal evolution of the transverse cross section of the pattern along the line indicated by the dashed arrow showing the nondrifting of the pattern. $h=14.6$, $\mu_{c} / \mu_{c}(h=0)=0.93$. The size of pictures (a) and (b) is, respectively, $1.3 \times 1.3 \mathrm{~mm}^{2}\left(130 \times 130 l_{d}^{2}\right)$ and $15 \times 15 \mathrm{mrad}\left(1.77 \times 1.77 l_{d}^{-2}\right)$. 

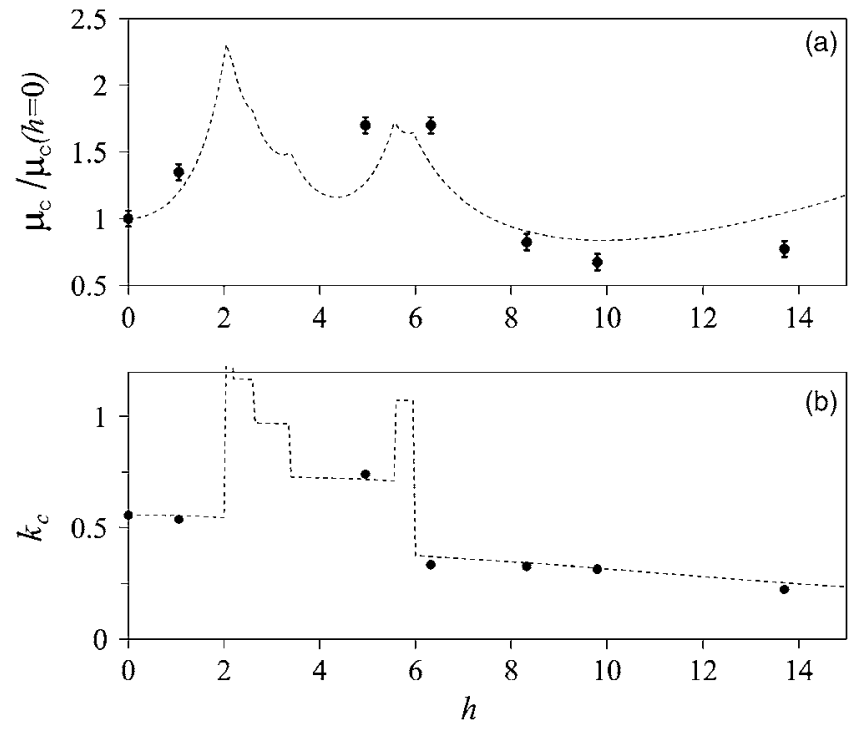

FIG. 17. Experimental evolution of the (a) convective threshold and (b) wave number of vertical rolls vs $h$. The continuous lines are the theoretical values calculated from the analytical expressions of Eqs. (5) and (11). Dots are the measured experimental data. Vertical bars are convective threshold determination errors. $\sigma=-15$.

edge [1]. The agreement between the wave-number analytical curves determined for the equivalent uniform system and the experimental values (dots) measured for the real system pumped with a Gaussian beam input is excellent [Fig. 17(b)]. Concerning the convective threshold [Fig. 17(a)], the agreement is also good, taking into account the presence of a Gaussian profile for the pumping beam that induces shifts in the threshold values. It is also clear here that because of convective instability, it is possible to lower by $10 \%$ the first threshold of pattern instability as compared to the absolute threshold in the absence of nonlocality $(h=0)$ for negative values of $\sigma$ (or equivalently for negative feedback lengths) as already mentioned by Ramazza et al. [19,26].

To summarize our experimental observations, we have obtained an excellent agreement between the predictions and the experimental observations on all the points that have been checked. More specifically, the nature of the modes predicted at the primary threshold, the variation with $h$ of their threshold $\mu_{c}(h)$ and wave number $k_{c}$, and their phase velocity $v_{\phi}$ agree in all details. This confirms that the model [Eqs. (2) and (3)] accurately describes pattern formation of our nonlocal system and generalizes what was obtained in the $1 \mathrm{D}$ configuration [1]. In these conditions, we can safely assume that the theoretical and numerical determinations of the absolute thresholds are also reliable. Unfortunately, no experimental check of absolute threshold is available for these 2D instabilities, in particular because of the possible interactions of several convective modes, contrary to the 1D configuration, where the evolution of the instability front $u_{f}$ provided such a check. Therefore, in the 2D case, we have to rely on the predicted evolutions and values of $\mu_{a}$. As the calculations of the absolute thresholds $\mu_{a}$ predict wide regions of purely convective regimes, i.e., domains in which $\mu_{c}$ and $\mu_{a}$ are significantly different, we can expect corresponding experimental large regions of purely convective patterns. Numerical simulations have also confirmed that the finite transverse dimension and Gaussian dependence of the pump transverse profile do not change significantly the relative values of the thresholds if the aspect ratio $\eta$ is large, which is the case here since $\eta \approx 30$. As simulations carried out with corresponding parameters confirm that structures disappear in the absence of noise, we can safely claim that the patterns obtained experimentally in these conditions just above the primary convective threshold are noise-sustained structures.

\section{CONCLUDING REMARKS}

In conclusion, we have shown that introducing a transverse shift in a feedback loop of a noisy optical system leads to convective and absolute instabilities generating noisesustained and dynamically self-sustained transverse structures, respectively. The first ones are convective patterns that would not be observed if there were no noise in the system. The second ones are the well known patterns that asymptotically cover the whole transverse space even in the absence of noise.

We have analytically predicted, in the ideal situation of a uniform incident wave, three types of "basic convective patterns" or "convective modes," namely horizontal rolls, vertical rolls, and rectangular lattices. Numerical simulations carried out for realistic experimental conditions confirm this analytical study and agree very well with the obtained experimental noise-sustained modes.

We have also experimentally evidenced the properties and features of the "convective modes" appearing in our optical system. In particular, horizontal rolls and rectangular lattices are purely noise-sustained structures and are nondrifting at convective threshold. Their related wave number and convective threshold do not evolve with the transverse shift $h$ that controls the nonlocality. We have also shown that the vertical rolls can be either noise-sustained or self-sustained and drift at convective threshold except for very specific values of $h$.

Numerous more complex patterns that can be generated from the basis of these three previous "convective modes" depending on their domains of existence and their competition are now under study. 
[1] E. Louvergneaux, C. Szwaj, G. Agez, P. Glorieux, and M. Taki, Phys. Rev. Lett. 92, 043901 (2004).

[2] R. J. Briggs, Electron-Stream Interaction with Plasmas (MIT Press, Cambridge, MA, 1964).

[3] P. Huerre and P. A. Monkewitz, Annu. Rev. Fluid Mech. 22, 473 (1990)

[4] A. Couairon and J. M. Chomaz, Phys. Rev. Lett. 79, 2666 (1997); H. R. Brand, R. J. Deissler, and G. Ahlers, Phys. Rev. A 43, 4262 (1991).

[5] M. Santagiustina, P. Colet, M. San Miguel, and D. Walgraef, Phys. Rev. Lett. 79, 3633 (1997).

[6] N. Mitarai and H. Nakanishi, Phys. Rev. Lett. 85, 1766 (2000).

[7] N. Israeli, D. Kandel, M. F. Schatz, and A. Zangwill, Surf. Sci. 494, L735 (2001).

[8] P. Büchel and M. Lucke, Phys. Rev. E 61, 3793 (2000); X. Nicolas, A. Mojtabi, and J. K. Platten, Phys. Fluids 9, 337 (1997).

[9] P. Gondret, P. Ern, L. Meignin, and M. Rabaud, Phys. Rev. Lett. 82, 1442 (1999).

[10] L. Pastur, M. T. Westra, and W. van de Water, Physica D 174, 71 (2003).

[11] P. L. Ramazza, S. Ducci, and F. T. Arecchi, Phys. Rev. Lett. 81, 4128 (1998); S. Ducci, P. L. Ramazza, W. González-Viñas, and F. T. Arecchi, ibid. 83, 5210 (1999).

[12] For a review of the different types of transverse nonlocal interactions in the feedback system and associated references, see Sec. 3.6. in F. T. Arecchi, S. Boccaletti, and P. L. Ramazza, Phys. Rep. 318, 1 (1999).

[13] P. A. Sturrock, Amplifying and Evanescent Waves, Convective and Non-convective Instabilities, edited by J. E. Drummond (McGraw-Hill, New York, 1961).
[14] C. Bender and S. Orszag, Advanced Mathematical Methods for Scientists and Engineers (McGraw-Hill, New York, 1978).

[15] S. A. Akhmanov, M. A. Vorontsov, and V. Yu. Ivanov, JETP Lett. 47, 707 (1988); S. A. Akhmanov, M. A. Vorontsov, V. Yu. Ivanov, A. V. Larichev, and N. I. Zheleznykh, J. Opt. Soc. Am. B 9, 78 (1992).

[16] W. J. Firth, J. Mod. Opt. 37, 151 (1990); G. D’Alessandro and W. J. Firth, Phys. Rev. Lett. 66, 2597 (1991).

[17] P. N. McGraw and M. Menzinger, Phys. Rev. E 72, 026210 (2005).

[18] G. Agez, C. Szwaj, E. Louvergneaux, and P. Glorieux, Phys. Rev. A 66, 063805 (2002).

[19] P. L. Ramazza, P. Bigazzi, E. Pampaloni, S. Residori, and F. T. Arecchi, Phys. Rev. E 52, 5524 (1995).

[20] A. D. D. Craik, Proc. R. Soc. London, Ser. A 373, 457 (1981); R. Tagg, W. S. Edwards, and H. L. Swinney, Phys. Rev. A 42, 831 (1990).

[21] See Sec. 4 in P. M. Morse and H. Feshbach, Methods of Theoretical Physics I (McGraw-Hill, New York, 1953).

[22] F. Papoff and R. Zambrini, Phys. Rev. Lett. 94, 243903 (2005); R. Zambrini and F. Papoff, Phys. Rev. E 73, 016611 (2006).

[23] H. Ward, M. N. Ouarzazi, M. Taki, and P. Glorieux, Phys. Rev. E 63, 016604 (2001); H. Ward, M. Taki, and P. Glorieux, Opt. Lett. 27, 348 (2002).

[24] E. Louvergneaux, Phys. Rev. Lett. 87, 244501 (2001).

[25] G. Agez, P. Glorieux, C. Szwaj, and E. Louvergneaux, Opt. Commun. 245, 243 (2005).

[26] P. L. Ramazza, S. Boccaletti, A. Giaquinta, E. Pampaloni, S. Soria, and F. T. Arecchi, Phys. Rev. A 54, 3472 (1996). 